\title{
A Influência da Nutrição Adequada e da Prática de Atividades Física na Saúde dos
}

\section{Adolescentes}

The Influence of Proper Nutrition and of Physical Activity Practice at Adolescent's Health

La Influencia de la Nutrición Adecuada y de la Práctica de la Actividad Física em la Salud de los Adolescentes

Carolina Júnia Reis Paz ${ }^{1}$

Kellen Bruna de Sousa Leite ${ }^{2}$

Nilcinádia Alves dos Anjos ${ }^{3}$

Ludmila Cotrim Fagundes ${ }^{4}$

Maria Fernanda Neves Silveira de Souza ${ }^{5}$

Daniel Antunes Freitas ${ }^{6}$

\section{Resumo}

Objetivo: Analisar a produção científica acerca da influência de uma nutrição adequada e da prática de atividades físicas na saúde do adolescente. Método: Revisão sistemática de literatura em outubro de 2016, com vista a responder a questão norteadora: "Qual a influência de uma nutrição adequada e da prática de atividades físicas na saúde do adolescente?". As bases de dados pesquisadas foram Science Direct, PUBMED e Scientific Electronic Library Online (SciELO/LILACS), utilizando-se os seguintes descritores: Nutrição, Adolescentes e Atividade Física. Foram incluídos artigos em Português, Inglês e Espanhol com textos disponíveis na íntegra e de livre acesso, publicados entre 2011 e 2016. Excluíram-se os artigos cuja temática destoou do objetivo pretendido e os repetidos. Resultados: Dos 15.202 artigos encontrados na pesquisa inicial,

${ }^{1}$ Graduanda do curso de Medicina na Faculdade Estadual de Montes Claros. Autor correspondente: Avenida Dr. Ruy Braga, S/N - Vila Mauriceia. CEP 39401-089. Montes Claros - MG. E-mail: carolinajrpaz@gmail.com

2,3,4,5 Graduanda em Medicina. Faculdade Estadual de Montes Claros.

${ }^{6}$ Doutor em Ciências da Saúde. Universidade Estadual de Montes Claros, Unimontes. Montes Claros, MG, Brasil.

Recebido: Dez/2016 - Aceito: Fev/2017. 
50 foram selecionados para compor os dados do trabalho. Conclusão: Diante do aumento das patologias relacionadas à alimentação faz-se necessário reforçar a importância da união de alimentação adequada e prática de atividade física regular, a fim de que se evite o início precoce de patologias relacionadas às dietas hipercalóricas, cada vez mais prevalentes entre os adolescentes.

\section{Descritores: Nutrição em Saúde Pública; Adolescente; Atividade Motora.}

\section{Abstract}

Objective: Analyze scientific production about in the influence of proper nutrition and physical activity in the adolescent's health. Methodology: Systematic review of literature, performed in October of 2016, in order to answer the guiding question: "What is the influence of a proper nutrition and of physical activity practice in adolescent's health?". The virtual databases researched were Science Direct, PUBMED e Scientific Electronic Library Online (SciELO/LILACS), using the descriptors: Nutrition, Adolescent and physical activity. Were included articles in Portuguese,
English and Spanish and with full free texts available, published between 2011 and 2016. Repeated articles and those without concordance with the proposed objective were excluded. Results: From 15.202 articles found in the first search, 50 were selected to compose the work data. Conclusion: In view of the increase in food-related pathologies, it is necessary to reinforce the importance of combining adequate diet and regular physical activity, in order to avoid the early onset of pathologies related to hypercaloric diets, which are increasingly prevalent among adolescents.

Descriptors: Nutrition, Public Health; Adolescent; Motor Activity.

\section{Resumen}

Objetivos: Analizar la producción científica sobre la influencia de la nutrición adecuada y la actividad física em la salud de los adolescentes. Método: Literatura revisión sistemática em octubre el ano, com el fin de responder a la pregunta de orientación: “ Cuál es la influencia de la nutricion adequada y la actividad física en la salud de lo adolescente?", desde la verificación de las bases de datos Science Direct, PUBMED e Scientific 
Electronic

Library

Online

(SciELO/LILACS), usando las seguientes palavras clave: Nutrición, Actividad Física y Adolescentes. Se incluyeron artículos en português, inglés y espanhol com textos disponibles en el acceso pleno y libre, publicados entre 2011 y 2016. Se excluyeron los artículos cuyo tema no estuvo de acuerdo cuando se compara el objetivo previsto y los repetidos. Resultados: De los 15.202 artículos que se encuentran em la búsqueda inicial, 50 fueron selecionados para componer los datos del trabajo. Conclusion: Ante el aumento de las enfermedades relacionadas con la alimentación, es necesario reforzar la importancia de la unión nutrición adecuada y la actividad física regular, a fin de evitar la aparición temprana de enfermedades relacionadas con la dieta alta en grasas, cada vez más frecuente entre los adolescentes.

\section{Descriptores: Nutrición en Salud} Pública; Adolescente; Actividad

Motora.

\section{Introdução}

De acordo com a Organização Mundial de Saúde a adolescência compreende a faixa etária entre os dez e dezenove anos de idade $^{(1)}$. Nesse momento de transição da infância para a idade adulta ocorre o desenvolvimento da independência onde o adolescente busca autonomia emocional, social e cognitiva $^{(2,3)}$. Além disso, ocorre o processo de maturação biológica, a puberdade, acarretando modificações quanto à imagem corporal e a sexualidade $^{(3)}$.

No contexto geral, uma nutrição adequada com a ingestão de nutrientes como fibras, vitaminas e minerais em quantidades suficientes é necessária para o funcionamento normal do corpo humano e evitar diversas doenças ${ }^{(4)}$. $\mathrm{O}$ consumo regular de frutas e verduras está associado à redução da ocorrência de doenças crônicas e do risco de mortalidade, além de prevenir deficiências de micronutrientes ${ }^{(5)}$. Por outro lado, a preferência por consumir alimentos ricos em gorduras e açúcares atua no sentido oposto aumentando a incidência dessas doenças ${ }^{(4)}$.

Atividade física pode ser definida como qualquer movimento corporal proporcionado pela musculatura esquelética resultando em gasto energético acima dos níveis de repouso $^{(6)}$. A prática regular de atividades físicas em intensidade adequada proporciona bem-estar e qualidade de vida e, assim como a 
nutrição, está relacionada com a diminuição do risco de doenças crônicas e de mortalidade $\operatorname{precoce}^{(7)}$.

A adolescência é um período crítico para o desenvolvimento e para o estabelecimento de comportamentos e atitudes que tendem a ser mantidos na vida adulta $^{(8)}$. Dessa forma, é essencial que esse grupo desenvolva hábitos como a ingestão balanceada de alimentos para satisfazer as necessidades nutricionais aumentadas nesse período $^{(1)}$ e proporcionar desenvolvimento adequado ${ }^{(9)}$.

Somado a nutrição equilibrada é importante que o adolescente realize a prática regular de atividades físicas a fim de adotar um estilo de vida saudável prevenindo o aparecimento de doenças crônicas. Além desses benefícios a realização de atividades físicas também está relacionada com aumento da aptidão física e redução dos sintomas de depressão ${ }^{(9)}$.

O objetivo do presente estudo é descrever os benefícios da nutrição adequada e da prática regular de atividades físicas para saúde do adolescente.

\section{Método}

Compreende em uma revisão integrativa da literatura, realizada por meio de pesquisa e avaliação criteriosa de estudos divulgados sobre a hipótese proposta. A avaliação dos resultados encontrados possibilita a execução das evidências abordadas na prática. A questão norteadora definida para a investigação do estudo foi "Qual a influência de uma nutrição adequada e da prática de atividades físicas na saúde do adolescente?".

A partir disso, foram requeridas as bases de dados virtuais: Science Direct, PUBMED e Scientific Electronic Library Online (SciELO/LILACS).

A procura por estudos nesta revisão literária foi mediante a combinação de três descritores, aplicando-se moduladores booleanos, indexados no DeCS (Descritores em Ciências da Saúde) recorrendo-se a linguagem única na indexação de artigos de revistas científicas, livros, anais de congressos, relatórios técnicos, e outros tipos de materiais, assim como a pesquisas e recuperação de assuntos da literatura científica. Foram usadas três combinações de descritores, procurados nos idiomas Inglês, Português e Espanhol. Para análise, os descritores foram utilizados conforme estão apresentados na Tabela 1. 
Tabela 01. Descritores Utilizados na Busca

\begin{tabular}{ccc}
\hline $\begin{array}{c}\text { Descritores em } \\
\text { Inglês }\end{array}$ & $\begin{array}{c}\text { Descritores em } \\
\text { Português }\end{array}$ & Descritores em Espanhol \\
\hline Nutrition And & Nutrição And Adolescente & Nutrición And Adolescente \\
Adolescent & Nutrição And Adolescente & Nutrición And Adolescente \\
Nutrition And & And Atividade física & And Actividad motora \\
Adolescent And Motor & & \\
activity & Atividade física And & Actividad motora And \\
Motor activity And & Adolescente & Adolescente \\
Adolescent & &
\end{tabular}

A interpretação dos dados seguiu critérios de inclusão baseados no tema proposto. Os critérios adotados para a inclusão de estudos nesta revisão sistemática foram estudos realizados entre os anos 2011 e 2016; de texto completo disponível online e referentes à nutrição e prática de atividades físicas entre adolescentes. Excluindo-se estudos que fugissem dos critérios de seleção previamente estabelecidos.

A fim de analisar qualitativamente os artigos encontrados na amostra final, utilizou-se os níveis de evidência propostos por Stillwell ${ }^{(10)}$ : I - Revisão sistemática ou metanálise; II - Ensaio clínico randomizado controlado; III Ensaio clínico controlado sem randomização; IV - Caso controle ou estudo de coorte; V - Revisão sistemática de estudo qualitativo ou descritivo; VI - Estudo qualitativo ou descritivo; VII - Artigo de opinião ou consenso de órgãos governamentais ou conselho de especialidades médicas. Os dados obtidos após leitura dos resumos e da leitura dos artigos foram apresentados na forma de resumo descritivo em tabelas e quadros.

\section{Resultados}

Após a revisão dos trabalhos, foi selecionado um total de 50 artigos, aplicados os critérios de inclusão e exclusão relatados. A primeira etapa da triagem selecionou um total de 309 artigos, escolhidos de uma quantidade inicial de 15202, com base na leitura dos títulos. Posteriormente, realizou-se a leitura dos resumos apresentados nos artigos selecionados, escolhendo-se 157 trabalhos que foram analisados 
integralmente. Ao final, restou uma amostra de 50 artigos.

De acordo com a tabela 2, do total de 50 artigos selecionados, $8,00 \%$ são do ano de $2011,12,00 \%$ são do ano de 2012, 12,00\% são do ano de 2013, $16,00 \%$ são do ano de 2014 e cada um dos anos de 2015 e 2016 representou $26,00 \%$ dos artigos.

Tabela 02. Números de Artigos de Acordo com o Ano de Publicação

\begin{tabular}{ccc}
\hline Ano de publicação & $\mathrm{n}$ & $\%$ \\
\hline 2011 & 4 & 8,00 \\
2012 & 6 & 12,00 \\
2013 & 6 & 12,00 \\
2014 & 8 & 16,00 \\
2015 & 13 & 26,00 \\
2016 & 13 & 26,00 \\
Total & 50 & 100
\end{tabular}

De acordo com a tabela 3 , dentre esses artigos da amostra final, 6,00\% correspondem ao primeiro nível de evidência (revisão sistemática), 2,00\% correspondem ao segundo nível de evidência (ensaio controlado randomizado) e ao terceiro nível de evidência (ensaio controlado não randomizado), 8,00\% correspondem ao quarto nível de evidência (coorte ou caso-controle), $16,00 \%$ correspondem ao quinto nível de evidência (revisão sistemática de estudo qualitativo ou descritivo), 66,00\% correspondem ao sexto nível de evidência (estudo qualitativo ou descritivo) e $0 \%$ correspondem ao sexto nível de evidência (artigo de opinião ou consenso de órgãos governamentais ou conselho especialidades médicas). 
Tabela 03. Artigos Distribuídos de Acordo com o Nível de Evidência

\begin{tabular}{cccc}
\hline Delineamento & Nível de evidência & $\mathrm{n}$ & $\%$ \\
\hline Revisão Sistemática & I & 3 & 6,00 \\
Ensaio Controlado Randomizado & II & 1 & 2,00 \\
Ensaio Controlado Não Randomizado & III & 1 & 2,00 \\
Caso Controle ou Estudo de Coorte & IV & 4 & 8,00 \\
Revisão Sistemática de Estudo & V & 8 & 16,00 \\
Qualitativo ou Descritivo & & 33 & 66,00 \\
Estudo Qualitativo ou Descritivo & VI & 0 & 0 \\
Artigo de Opinião ou Consenso de & & & \\
Órgãos Governamentais ou Conselho & VII & & \\
de Especialidades Médicas & & 50 & 100 \\
\hline Total & & & \\
\hline
\end{tabular}

\section{Discussão}

Durante as análises, percebeu-se que muitos adolescentes mantêm uma baixa ingestão de alimentos suficientemente nutritivos e uma exacerbada realização de exercícios físicos devido a uma tentativa de adequar-se a um padrão de beleza ditado pela sociedade, práticas essas altamente nocivas ao seu estado de saúde $^{(11-12)}$. Estudos comprovam que o tipo de alimento ingerido está intimamente relacionado com padrões corporais, ou seja, a dieta adequadamente nutritiva, baseada em arroz e feijão, é mais utilizada por indivíduos eutróficos ${ }^{(13-14)}$, enquanto alimentos de fácil preparo, altamente calóricos e com menores cargas nutricionais, costumam ser mais consumidos por pessoas com sobrepeso e obesidade ${ }^{(13-15)}$.

O costume dos adolescentes de se alimentar em família promove uma melhor ingestão nutricional devido a uma eficiente organização das refeições, cada uma com as adequadas quantidades de micro e macronutrientes necessárias ${ }^{(16-18)}$. Por outro lado, o hábito de comer na frente de telas (computador e televisão) e não à mesa, em família, aumenta o consumo de alimento gordurosos e pouco nutritivos, como fast food, refrigerantes etc, além do aumento do sedentarismo ${ }^{(19-22)}$. 
É extremamente importante que um estilo de vida saudável seja desenvolvido no período da adolescência, porque os hábitos desenvolvidos nessa fase são, na maioria dos casos, perpetuados ao longo da vida ${ }^{(23,24)}$. É baixa a prevalência de adolescentes que praticam atividade física suficiente para resultar em benefícios $(60 \mathrm{~min} / \mathrm{dia}$ de atividade física moderada a vigorosa) em um número elevado de países, incluindo o Brasil, contribuindo para o aumento de doenças não transmissíveis ${ }^{(23,25)}$. Nesse âmbito, as mulheres são apontadas como as mais sedentárias devido a fatores não conhecidos, sugestionandose para fatores psicológicos, culturais e comportamentais $^{(23,26,27)}$. A partir do Modelo Transteorético (MT), que classifica os indivíduos em cinco estágios de mudanças de comportamento $(\mathrm{EMC})$ em relação à atividade física: pré-contemplação, contemplação, preparação, ação e manutenção, sugerem uma prevalência do sexo feminino maior nos estágios iniciais e o oposto acontece para o sexo masculino $^{(28)}$. Em ambos os sexos, os níveis da prática de atividade física tendem a diminuir com a idade, esse declínio parece estar mais associado a idade biológica (progressão para o estado de maturidade) do que a idade cronológica $^{(27)}$.

Um exemplo de doenças não transmissíveis causadas pela má nutrição e inatividade física é a obesidade, que é o transtorno nutricional de maior frequência entre os jovens e está mais prevalente na área urbana e entre adolescentes que tiveram em sua infância um contexto familiar carente de hábitos alimentares adequados e estilo de vida ativo(29), sendo considerada uma pandemia atual com grandes custos para os sistemas de saúde no mundo todo ${ }^{(30)}$. Dentre as causas da obesidade, está a alta ingestão calórica, responsável pelas alterações lipídicas, geralmente mantidas durante a

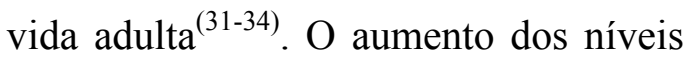
lipídicos, principalmente na região abdominal, bem como a baixa ingestão de micronutrientes têm se mostrado importantes fatores de propensão a patogenias $^{(31-33,35-37)}$.

Algumas dessas patogenias são a hipertensão arterial sistêmica, que aumenta o risco de problemas cardiovasculares $^{(24,38)}$ e transtornos endócrinos metabólicos, como a dislipidemia e resistência insulínica ${ }^{(24,39)}$, que aumentam o risco de incidência de diabetes mellitus tipo II. Apneia do sono, osteoartrites, certos tipos de câncer e vários problemas 
psicológicos também são consequências da obesidade crônica ${ }^{(40)}$. Todas essas patologias eram próprias da idade adulta, porém estão acometendo, cada vez mais, os jovens ${ }^{(24)}$. Essa informação tem grande relevância, já que a obesidade, quando surge e se agrava na adolescência, torna-se mais problemática por ser essa uma fase de intensas transformações, crescimento e desenvolvimento ${ }^{(40)}$. Vale ressaltar que a atividade física na prevenção da obesidade é mais significativa do que no seu tratamento ${ }^{(41)}$.

A prevalência maior da obesidade na área urbana, citada anteriormente, pode estar associada a fatores como violência, que limita a prática de atividades físicas na rua. Desse modo, atividades cotidianas e brincadeiras que eram praticadas na rua são substituídas por atividades sedentárias ${ }^{(42)}$. Além disso os avanços tecnológicos favorecem estilos de vida sedentários, a exemplo o uso exagerado de games e televisão ${ }^{(42,43)}$, sendo esta também um veículo de divulgação de alimentos altamente calóricos ${ }^{(42)}$. Ademais, a inatividade física está associada com o consumo inadequado de frutas e vegetais $^{(7)}$.

Outro fator que está relacionado com a adoção de uma rotina sedentária é o isolamento social entre os adolescentes. Pesquisas relatam que jovens que têm uma relação social fora do horário escolar realizam mais exercícios físicos ${ }^{(44)}$. Isso pode ser explicado porque o apoio recebido pela família, amigos e professores é fundamental para a adoção de prática de atividades físicas ${ }^{(24,25,45)}$. Esse apoio social apresenta duas categorias, uma que está ligada a aspectos tangíveis, a exemplo fatores econômicos que incluem pagamento de mensalidades e transporte, bem como a aquisição de equipamentos; e outra que está relacionada com aspectos intangíveis, a exemplo o encorajamento, o incentivo e os elogios ${ }^{(24)}$. Além disso, estudos sugerem que a baixa competência motora, que pode ser definida como a capacidade de realizar atividades físicas em seus variados graus, tem sido relacionada com a menor prática de exercícios entre os jovens ${ }^{(46)}$.

Tendo em vista as problemáticas na saúde que a baixa atividade física ocasiona nos adolescentes, escolas devem ser o alvo de intervenções para melhorar a prática de exercícios nessa faixa etária ${ }^{(44,47,48)}$, sendo as aulas de educação física um cenário ideal nesse processo $^{(44)}$. Para o planejamento das intervenções, é preciso entender os fatores que estão associados à prática de atividade física, sendo eles sociais, 
ambientais, pessoais, físicos ${ }^{(7,25,26,47)}$, demográficos e psicológicos ${ }^{(25,26)}$. Aumentar a prática de atividade física e induzir uma alimentação saudável entre os jovens são importantes, porque trazem benefícios para a saúde, como a redução de riscos cardiovasculares, controle e manutenção do peso corporal $^{(40,49)}$, melhora da força muscular e do desempenho acadêmico $^{(25,48)}$ bem como aumenta as chances dos indivíduos manterem-se ativos durante a vida adulta ${ }^{(25,26,30,47)}$. Visto que a depressão é um dos problemas de saúde de maior importância atualmente, é fundamental também se fazer associação entre atividade física e saúde mental, já que a inatividade física aumenta a predisposição a depressão ${ }^{(50)}$.

\section{Conclusão}

A nutrição e a prática de atividades físicas representam importantes fatores de influência no desenvolvimento da saúde dos adolescentes. Apesar da existência de uma exacerbada preocupação dessa faixa etária com a manutenção da aparência física ideal, dependente de exageradas dietas hipocalóricas e práticas físicas, a maior prevalência no que se refere aos desarranjos desses fatores na saúde juvenil ainda é representada pelas dietas hipercalóricas e pelo sedentarismo, cada vez mais presentes. A partir dessa constatação, depara-se com uma preocupante realidade, representada pela prematurização de patologias relacionadas a esse problema, antes caracterizadas pelo maior acometimento de adultos.

\section{Referências}

1. Teji K, Dessie Y, Assebe T, Abdo $\mathrm{M}$. Anaemia and nutritional status of adolescent girls in Babile District, Eastern Ethiopia. Pan Afr. Med. J. [online journal]. 2016 [cited 2016 oct. 03]; 24(62). Available from: https://www.ncbi.nlm.nih.gov/pmc/arti cles/PMC5012790.

2. Raimundi MJ, Molina MF, Gimenez M, Minichiello C. ¿Qué es un desafío? Estudio cualitativo de su significado subjetivo en adolescentes de Buenos Aires. Rev. latinoam. cienc. soc. niñez juv. [periódico en Internet]. 2014 [citado 2016 oct. 03]; 12(2):521-34. Disponible en: http://www.scielo.org. co/scielo.php? script $=$ sci_arttext\&pid $=\mathrm{S}$ 1692-715X2014000200002\&lng=en.

3. López-Navarrete GE, Perea-Martínez A, Padrón-Martínez MM, EspinozaGaramendi E, Lara-Campos A. Entrevista con el adolescente. Acta Pediátr. Mex. [periódico en Internet]. 2014 [citado 2016 oct. 03]; 35(3):22937. Disponible en: http://www.scielo. org.mx/scielo.php?script=sci_arttext\&pi $\mathrm{d}=\mathrm{S} 0186-23912014000300009$. 
4. Pereira RC, Santana ML, Luna TPA, Corrêa MSM, Santos ACO. Food Intake, Nutritional Status And Socioeconomic Profile of Women Attending a Public Service. Rev. enferm. UFPE on line. [online journal]. 2014 [cited 2016 oct. 03]; (9)26. Available from: http://www.revista.ufpe .br/revistaenfermagem/index.php/revista /article/view/6273/pdf_6101.

5. Santana JT, Furtado MB, Pinho L. Promotion of Fruit And Vegetable Consumption in Adolescents: Case Studies. Rev. enferm. UFPE on line [online journal]. 2014 [cited 2016 oct. 03]; 8(1). Available from: http://www.revista.ufpe.br/revistaenfer magem/index.php/revista/article/view/5 566/pdf_4491.

6. Polisseni MLC, Ribeiro LC. Exercício físico como fator de proteção para a saúde em servidores públicos. Rev. bras. med. Esporte [periódico na Internet]. 2014 [citado 2016 out. 03]; 20(5):340-4. Disponível em: http://www .scielo.br/scielo.php?script=sci_arttext\& pid $=$ S1517-86922014000500340\&lng $=$ em.

7. Silva DAS, Silva RJS. Association between physical activity level and consumption of fruit and vegetables among adolescents in northeast Brazil. Rev. paul. pediatr. [online journal]. 2015 [cited 2016 oct. 03]; 33( 2 ):16773. Available from: http://www. scielo.br/scielo.php?script $=$ sci_arttext\& pid $=$ S0103-05822015000200167\&lng= en.

8. SILVA MP, Guimarães RF, Mazzardo O, Martins RV, Watanabe PI, Campos W. Atividade Física e Agregação de Fatores de Risco Metabólicos em Adolescentes. Rev. educ. fis. [periódico na Internet]. 2015 [citado 2016 out. 03]; 26(4):611-9. Disponível em: http://www.scielo.br/ scielo.php?script $=$ sci_arttext\&pid $=\mathrm{S} 198$ 3-308320150004006 1 1\&lng=pt\&nrm=i so.
9. Straatmann VS, Oliveira AJ, Rostila $\mathrm{M}$, Lopes CS. Changes in physical activity and screen time related to psychological well-being in early adolescence: findings from longitudinal study ELANA. BMC public health [online journal]. 2016 [cited 2016 oct. 03]; 16:977. Available from: https://www.ncbi.nlm.nih.gov/pubmed/ 27630121

10. Stillwell SB, Fineout-Overholt E, Melnyk BM, Williamson KM. Searching for the Evidence: Strategies to help you conduct a successful search. Aust j. adv. nurs. [online journal]. 2010 [cited 2016 oct. 03]; 110(5). Available from: http://www.nursingcenter.com/ nursingcenter_redesign/media/ebp/ajnse ries/searching.pdf.

11. Bissochi CO, Juzwiak CR. Avaliação nutricional e da percepção da autoimagem corporal de atletas adolescentes de voleibol. Nutrire Rev. Soc. Bras. Aliment. Nutr. [periódico na Internet]. 2012 [citado 2016 out. 04]; 37(1):34-53. Disponível em: http://bases .bireme.br/cgi-bin/wxislind.exe/iah/onli ne/?IsisScript $=\mathrm{iah} / \mathrm{iah} . \mathrm{xis} \& \mathrm{src}=$ google $\&$ base $=$ LILACS\&lang $=p \&$ nextAction $=1 n$ $\mathrm{k} \&$ exprSearch $=658483$ \&indexSearch $=\mathrm{I}$ D.

12. Fortes LS, Morgado FFR, Ferreira MEC. Fatores associados ao comportamento alimentar inadequado em adolescentes escolares. Rev. psiquiatr. clín. [periódico na Internet]. 2013 [citado 2016 out. 04]; 40(2):59-64. Disponível em: http://www.scielo.br/ scielo.php?script=sci_arttext\&pid=S010 $1-60832013000200002 \& \operatorname{lng}=\mathrm{en}$.

13. Salvatti AG, Escrivão MAMS, Taddei JAAC, Bracco MM. Padrões alimentares de adolescentes na cidade de São Paulo. Rev. nutr. [periódico na Internet]. 2011 [citado 2016 out 04]; 24(5):703-13. Disponível em: http://www.scielo.br/scielo.php?script=s ci_arttext\&pid=S1415-5273201100050 0004\&lng=en. 
14. Rodrigues PRM, Pereira RA, Cunha DB, Sichieri R, Ferreira MG, Vilela AAF, et al. Fatores associados a padrões alimentares em adolescentes: um estudo de base escolar em Cuiabá, Mato Grosso. Rev. bras. epidemiol. [periódico na Internet]. 2012 [citado 2016 out. 04]; 15(3):662-74. Disponível em: http://www.scielo.br/scielo.php?script=s ci_arttext\&pid=S1415-790X201200030 0019\&lng $=\mathrm{en}$.

15. Souza AM, Barufaldi LA, Abreu GA, Giannini DT, Oliveira CL, Santos $\mathrm{MM}$, et al. ERICA: intake of macro and micronutrients of Brazilian adolescents. Rev. saúde pública [online journal] 2016 [cited 2016 oct. 04]; 50(1):1-15. Available from: http://www.scielo.br /scielo.php?script $=$ sci_arttext\&pid $=\mathrm{S} 00$ $34-89102016000200309 \& \operatorname{lng}=$ en.

16. Barufaldi LA, Abreu GA, Oliveira JS, Santos DF, Fujimori E, Vasconcelos SML, et al. ERICA: prevalence of healthy eating habits among Brazilian adolescents. Rev. saúde pública [online journal]. 2016 [cited 2016 oct. 04]; 50(1):1-9. Available from: http://www.scielo.br/scielo.php?script=s ci_arttext\&pid=S0034-8910201600020 0301\&lng=en.

17. Sánchez MJA, Díaz JAJ. Dietary risk behaviors among adolescents and young people of Nuevo Leon. Rev. Mex. de transtor. aliment. [online journal] 2015 [cited 2016 oct. 04]; 6:112. Available from: http://www.sciencedirect.com/science/a rticle/pii/S2007152315000099.

18. Banna JC, Buchthal OV, Delormier T, Kanashiro HMC, Penny ME. Influences on eating: a qualitative study of adolescents in a periurban area in Lima, Peru. BMC public health [online journal]. 2016 [cited 2016 oct. 04]; 16(40):1-11. Available from: https:/www.ncbi.nlm.nih.gov/pubmed/ 26772177.
19. Oliveira JS, Barufaldi LA, Abreu GA, Leal VS, Brunken GS, Vasconcelos SML, et al. ERICA: use of screens and consumption of meals and snacks by Brazilian adolescents. Rev. saúde pública [online journal]. 2016 [cited 2016 oct. 04]; 50(1):1-9. Available from: http://www.scielo.br/ scielo.php?script $=$ sci_arttext\&pid=S003 4-89102016000200302\&lng=en.

20. Bibiloni MM, Pich J, Córdova A, Pons A, Tus JÁ. Association between sedentary behaviour and socioeconomic factors, diet and lifestyle among the Balearic Islands adolescents. BMC public health [online journal]. 2012 [cited 2016 oct. 04]; 12:718. Available from: http://bmcpublichealth.biomedcen tral.com/articles/10.1186/1471-2458-12 -718 .

21. Powell LM, Nguyen BT. Fast-food and full-service restaurant consumption among children and adolescents: effect on energy, beverage, and nutrient intake. JAMA Pediatr. [online journal]. 2013 [cited 2016 oct. 04]; 167(1):14-20. Available from: https:/www.ncbi.nlm .nih.gov/pubmed/23128151.

22. Fletcher E, Leech R, McNaughton AS, Dunstan DW, Lacy KE, Salmon J. Is the relationship between sedentary behavior and cardiometabolic health in adolescents independent of dietary intake? A systematic review. Obes. rev. [online journal]. 2015 [cited 2016 oct. 04]; 16:795-805. Available from: https://www.ncbi.nlm.nih.gov/pubmed/ 26098509.

23. Barufaldi LA, Abreu GA, Coutinho ESF, Bloch KV. Meta-analysis of the prevalence of physical inactivity among Brazilian adolescents. Cad. Saúde Pública [online journal]. 2012 [cited $2016 \quad$ oct. 04];28(6):1019-32. Available from: http://www.scielosp .org/scielo.php?script $=$ sci_arttext\&pid= S0102-311X2012000600002\&lng=en. 
24. Prado CV, Lima AV, Fermino RC, Añez CRR, Reis RS. Apoio social e prática de atividade física em adolescentes da rede pública de ensino: qual a importância da família e dos amigos? Cad. Saúde Pública [periódico na Internet]. 2014 [citado 2016 out. 04] ; 30(4):827-38. Disponível em: http://www.scielo.br/scielo.php?script $=\mathrm{s}$ ci_arttext\&pid=S0102-311X201400040 0827.

25. Farias Júnior JC, Reis RS, Hallal PC. Physical activity, psychosocial and perceived environmental factors in adolescents from Northeast Brazil. Cad. Saúde Pública [online journal]. 2014 [cited 2016 oct. 04] ; 30(5):941-51. Available from: http://www.scielosp. org/scielo.php?script=sci_arttext\&pid= S0102-311X2014000500941\&lng=en.

26. Ardestani M, Niknami S, Hidarnia A, Hajizadeh E. Predictors of Physical Activity among Adolescent Girl Students Based on the Social Cognitive Theory. J. Res Health Sci [online journal]. 2015 [cited 2016 oct. 04]; 15(4):223-7. Available from: https://www.ncbi.nlm.nih.gov/pubmed/ 26728907

27. Bacil EDA, Mazzardo Júnior $O$, Rech CR, Legnani RFDS, Campos W. Physical activity and biological maturation: a systematic review. Rev. paul. pediatr. [online journal]. 2015 [cited 2016 oct. 04]; 33(1):114-21. Available from: http://www.science direct.com/science/article/pii/S0103058 214000276.

28. SILVA J, SILVA K. Estágios de mudança de comportamento para atividade física em adolescentes: revisão sistemática. Rev. bras. ativ. fis. saúde [periódico na Internet]. 2015 [citado 2016 out. 04]; 20(3):214. Disponível em: https://periodicos.ufpel .edu.br/ojs2/index.php/RBAFS/article/v iew/4613/4459.
29. González-Jiménez E, Cañadas GR, Lastra-Caro A, Fuent GAC. Efectividad de una intervención educativa sobre nutrición y actividad física en una población de adolescentes: Prevención de factores de riesgos endocrinometabólicos y cardiovasculares. Aquichan [periódico en Internet]. 2014 [citado 2016 oct. 04]; 14(4):549-59. Disponible en: http://www.redalyc.org /articulo.oa?id=74133057009.

30. Gordia AP, Quadros TMB, Silva LR, Santos GM. Knowledge of pediatricians regarding physical activity in childhood and adolescence. Rev. paul. pediatr. [online journal]. 2015 [cited 2016 oct. 04]; 33(4):400-6. Available from: http://www.scielo.br/ scielo.php?pid $=$ S0103-0582201500040 0006\&script $=$ sci_arttext\&tlng=pt.

31. Salvador CCZ, Kitoko PM, Gambardella AMD. Nutritional status of children and adolescents: factors associated to overweight and fat accumulation. Rev. bras. crescimento desenvolv. hum. [online journal]. 2014 [cited 2016 oct. 04]; 24(3):313-9. Available from: http://pepsic.bvsalud. org/scielo.php?script $=$ sci_arttext\&pid= S0104-12822014000300011\&lng=pt\&n $\mathrm{rm}=\mathrm{iso} \& \mathrm{t} \operatorname{lng}=\mathrm{en} \& O R I G I N A L L A N G=\mathrm{e}$ $\mathrm{m}$.

32. Souza JB, Enes CC. Influência do consumo alimentar sobre o estado nutricional de adolescentes de Sorocaba-SP. J. Health Sci. Inst. [periódico na Internet]. 2013 [citado 2016 out. 04]; 31(1):65-70. Disponível em: http://bases.bireme.br/cgi-bin/wxisl ind.exe/iah/online/?IsisScript=iah/iah.xi $\mathrm{s} \& \mathrm{src}=$ google $\&$ base $=$ LILACS\&lang $=\mathrm{p}$ \&nextAction $=$ lnk\&exprSearch $=684779$ \&indexSearch=ID. 
33. Dutra GF, Kaufmann CC, Pretto ADB, Albernaz EP. Sedentary lifestyle and poor eating habits in childhood: a cohort study. Ciênc. saúde coletiva [online journal]. 2016 [cited 2016 oct. 04]; 21(4): 1051-59. Available from: http://www.scielo.br/scielo.php?script $=$ s ci_arttext\&pid=S1413-8123201600040 1051 .

34. Lima-Serrano $\mathrm{M}$, Guerra-Martín MD, Lima-Rodríguez JS. Estilos de vida $\mathrm{y}$ factores asociados a la alimentacíon y la actividad física en adolescentes. Nutr. Hosp. [periódico en Internet]. 2015 [citado 2016 oct. 04]; 32(6):2838-47. Disponible en: http://www.redalyc.org/articulo.oa?id=3 09243321064.

35. Veiga GV, Costa RS, Araújo MC, Bezerra IN, Barbosa FS, Sichieri R, et al. Inadequate nutrient intake in Brazilian adolescents. Rev. saúde pública [online journal]. 2013 [cited 2016 oct. 04]; 47(1):212-21. Available from: http://www.scielo.br/scielo.php? script $=$ sci_arttext\&pid=S0034-8910201 $3000700007 \& \operatorname{lng}=$ en\&nrm $=$ iso\&tlng $=e$ $\mathrm{m}$.

36. Gómes WV, la Campa GLA, Reyes TMER, Tobar CFP. Estado nutricional en adolescentes, exceso de peso corporal y factores asociados. Rev. cuba. endocrinol. [periódico en Internet]. 2011 [citado 2016 oct. 04]; 22(3):225-36. Disponible en: http://scielo.sld.cu/scielo.php?script=sci arttext\&pid=S1561-295320110003000 05\&lng $=$ es.

37. Salam RAM, Hooda M, Das JK, Arshad A, Lassi ZS, Middleton P, et al. Interventions to Improve Adolescent Nutrition: A Systematic Review and meta-Analysis. J. adolesc. health [online journal]. 2016 [cited 2016 oct. 04]; 59:29-39. Available from: https://www .ncbi.nlm.nih.gov/pubmed/27664593.
38. Corrêa Neto VG, Palma A. Pressão arterial e suas associações com atividade física e obesidade em adolescentes: uma revisão sistemática. Ciênc. saúde coletiva [periódico na Internet]. 2014 [citado 2016 out. 04]; 19(3):797-818. Disponível em: http://www.scielosp.org/scielo.php?scri $\mathrm{pt}=$ sci_arttext\&pid=S1413-8123201400 0300797\&lng=en.

39. Múnera NE, Uscátegui RM, Parra BE, Manjarrés LM, Patiño F, Velásquez $\mathrm{CM}$, et al . Factores de riesgo ambientales y componentes del síndrome metabólico en adolescentes con exceso de peso. Biomédica [periódico en Internet]. 2012 [citado 2016 oct. 04]; 32(1):77-91. Disponible en: http://www.scielo.org.co/scielo.php ?script $=$ sci_arttext\&pid=S0120-415720 12000100010.

40. Campos LF, Almeida JZ, Campos FF, Campos LA. Prática alimentar e de atividade física em adolescentes obesos de escolas públicas e privadas. Rev. bras. promoç. saúde [periódico na Internet]. 2014 [citado 2016 out. 04]; 27(1):92-100. Disponível em: http://ojs.unifor.br/index.php/RBPS/arti cle/view/3163/pdf4.

41. Farias EDS, Gonçalves EM, Morcillo AM, Guerra-Júnior G, Amancio OMS. Effects of programmed physical activity on body composition in post-pubertal schoolchildren. J. pediatr. [online journal]. 2015 [cited 2016 oct. 04] 91(2):122-9. Available from: http://www.sciencedirect.com/ science/article/pii/S2255553615000208.

42. Dias PJP, Domingos IP, Ferreira MG, Muraro AP, Sichieri R, GonçalvesSilva RMV. Prevalence and factors associated with sedentary behavior in adolescents Rev. saúde pública [online journal]. 2014 [cited 2016 oct. 04] ; 48(2):266-74. Available from: http://www.scielo.br/scielo.php?script $=\mathrm{s}$ ci_arttext\&pid=S0034-8910201400020 0266\&lng=en\&nrm=iso\&tlng $=\mathrm{em}$. 
43. Jalali-Farahani S, Parisa A, e Yit S C. Are physical activity, sedentary behaviors and sleep duration associated with body mass index-for-age and health-related quality of life among high school boys and girls?. Health qual. life outocomes [online journal]. 2016 [cited 2016 oct. 04]; 14:30. Available from: https://www.ncbi.nlm.nih.gov/pmc/artic les/PMC4769527/.

44. Santos SJ, Hardman CM, Barros SSH, Santos CFBF, Barros MVG. Association between physical activity, participation in Physical Education classes, and social isolation in adolescents. J. pediatr. [online journal]. 2015 [cited 2016 Oct 04]; 91(6):54350. Available from: http://www.scielo .br/scielo.php?script $=$ sci_arttext\&pid $=\mathrm{S}$ 0021-75572015000600543\&lng=en.

45. Cheng LA, Mendonça G, Farias Júnior JC. Physical activity in adolescents: analysis of the social influence of parents and friends. J. pediatr. [online journal]. 2014 [cited 2016 oct. 04]; 90(1):35-41. Available from: http://www.scielo.br/scielo.php? pid $=$ S0021-75572014000100035\&script $=$ sci_arttext\&tlng=pt.

46. García CE, Pérez SJJ, Rodríguez GPL, Guillamón AR, López MPA, López VFJ. The relationship between segmental coordination, agility and physical activity in adolescents. Motriz rev. educ. fís. [online journal]. 2015 [cited 2016 oct. 04]; 21(2):200-06. Available from: http://www.scielo.br/ scielo.php?script $=$ sci_arttext\&pid $=\mathrm{S} 198$ 0-65742015000200200\&lng=en.

47. Azevedo MR, Menezes AM, Assunção MC, Gonçalves H, Arumi I, Horta BL, et al. Tracking of physical activity during adolescence: the 1993 Pelotas Birth Cohort, Brazil. Rev. saúde pública [online journal]. 2014 [cited 2016 oct. 04]; 48(6):925-30. Available from: http://www.scielosp.org/scielo. php?script $=$ sci_arttext\&pid=S0034-891 02014000600925.
48. Morton Kl, Atkin AJ, Corder K, Suhrcke M, Sluijs EMF. The school environment and adolescent physical activity and sedentary behaviour: a mixed-studies systematic review. Obes. rev. [online journal]. 2016 [cited 2016 oct. 04]; 17 (2): 142-58. Available from: http://onlinelibrary.wiley.com/doi/10.11 11/obr.12352/full.

49. Tarp J, Brond JC, Andersen LB, Moller NC, Froberg K, Grontved A .Physical activity, sedentary behavior, and long-term cardiovascular risk in young people: A review and discussion of the methodology in prospective studies. J. Esport Health Sci. [online journal]. 2016 [cited 2016 oct. 04]; 5(2):145-50. Available from: http://www.sciencedirect.com/science/a rticle/pii/S2095254616300035.

50. Poulsen $\mathrm{PH}$, Biering $\mathrm{K}$, Andersen $\mathrm{JH}$. The association between leisure time physical activity in adolescence and poor mental health in early adulthood: a prospective cohort study. BMC public health [online journal]. 2016 [cited 2016 oct. 04]; 16(1):1. Available from: http://bmcpublichealth .biomedcentral.com/articles/10.1186/s1 2889-015-2658-5. 